\title{
Studying the Interrelationship amongst various Performance Metrics for Third Party Logistic Service Providers in Construction Projects
}

\author{
S. P. Singh \\ Department of \\ Management Studies, IIT \\ Delhi, \\ Delhi, India
}

\author{
Viraj Voditel \\ Techture Structures \\ Private Limited \\ Nagpur, India
}

\author{
Remica Aggarwal \\ MIT-SOER, MIT-ADT \\ University, Pune, India
}

\author{
V. K. Aggarwal \\ Recventures Education \\ Services Private Limited \\ Delhi, India
}

\begin{abstract}
The paper focuses on the service oriented sector of third party logistic service provider. The objective of the paper is to first identify various key performance indicators or metrics to measure and assess the performance of supply chains of third party logistic service providers in construction projects and to further study the interrelationship amongst them using ISM methodology.
\end{abstract}

\section{Keywords}

Solid waste management ; ISM methodology ; Incineration, Solid waste, Hazardous, Non-hazardous

\section{INTRODUCTION}

The construction industry is a typical industry that works in temporary organizations, i.e. projects. It typically has an engineer to order setup where most of the products are physically big and immobile and consequently have to be produced on the spot (Vrijhoef and Koskela, 2000; Dubois and Gadde, 2002; Bakker, 2010 ). To deal with uncertain market situations and economic cycles diverse and dynamic client behavior; requirement of specialist manpower; large, heavy and immobile products (such as building tunnels , bridges etc. ) which require to be built on the construction etc. As much as 60-80 \% of the gross work done in construction projects involves the buying-in of materials and services from suppliers and subcontractors, leading to that these supply chain actors heavily impact the performance of construction projects (Dubois and Gadde, 2002, Miller et al., 2002). Hence, the construction supply chain is regarded as complex with interactions between multiple actors during the construction process (Winch, 2001).

Materials used in construction industry majorly falls into basic categories such as heavy materials (typically concrete, sand, gravel, bricks, timber, etc. ) ; light materials (which include structure completion and decorating materials (Agapiou et al., 1998b); standard materials (such as plasterboards or kitchen cabinets (Dubois and Gadde, 2000)); and project specific materials (prefabricated concrete elements and ventilation installations). These materials make up for a large part of the construction cost (Vidalakis and Sommerville, 2013). These material suppliers could be manufacturers and wholesalers or building merchants.

It is really hard for construction companies to keep pace with the client's demands of lower cost, higher quality, shorter duration of execution and more reliable schedules (Azambuja and O'Brien, 2009). At the same time, the set of TPL are temporary and fragmented. Logistics management in a construction supply chain is thus seen as a key competitive factor due to the importance of perfect deliveries towards construction sites. In the absence of failed or delayed deliveries to the construction site, quality and quantity of the potential customers decreases (Thunberg and Persson, 2014).

With the increased importance of logistics in construction, the evaluation of logistics effectiveness and efficiency is gaining increased attention. Logistics performance management is the key to quantify the current state and improvement potentials within logistics. A PMS, consisting of several KPIs, is seen as a key for creating transparency and a trigger for improvement ideas (Dörnhöfer et al., 2016). In the context of the construction industry, managing the communication and relationship with subcontractors will lead to less fragmented supply chains, greater control and focus on quality (Karim $e t$ al., 2006).

The characteristics of the temporary organization naturally also affect a construction project. In construction, time is of the essence and work is often done towards a deadline (which also can be interpreted as a goal). This makes effective planning an important and crucial skill to master in construction projects particularly when it is characterized by high uncertainty due to the lack of information. Furthermore, since much of the construction works are carried out outdoors which is quite obviously prone to weather conditions such as rain and snow. Weather and temperature will also affect the productivity of the construction workers. Supply chain management has been argued to increase the construction industry's low productivity and reduce the total cost (Vrijhoef and Koskela, 2000). It has also been argued that for SCM to be realized in construction there is a need for change driven by the construction clients. It is the client that procures the construction project and therefore the client has a critical role in the supply chain integration (Briscoe et al., 2004). Yet, due to the lack of in-house knowledge of SCM and logistics (Cox, 2008), contractors and clients have started to turn to thirdparty logistics (3PL) providers, especially for large construction projects. This is a new phenomenon not only for both the clients and the contractors but also for the 3PL or TPL providers not traditionally being very active in the construction industry. Also suppliers and transport providers serving the construction industry face new challenges with 3PL (Ekeskär et al., 2014; Lindén and Josephson, 2013).

A 3PL - short for third-party logistics (sometimes called a TPL) - is used in logistics and supply-chain management to outsource part or all of a business' distribution and fulfillment services. They can be transportation based (which specializes in the actual transport between locations - e.g., shipping); 
warehouse and distribution based (who handles storage, shipment, and returns) and financial and information based (who help to optimize the entire logistics network, owned and via third parties, freight auditing, cost accounting and control, and tools for monitoring, booking, tracking, tracing, and managing inventory) .

Tracing back the history of TPLs. During 1970s - 1980s, there is a rise of TPL as more companies chose to outsource transportation and logistics. During 1990s, there was a rise of technology and globalization revolutionized the supply chain environment. During 2008, there was a great recession which squeezed TPLs to a greater extent than ever before. From 2010 till present, near shoring and cloud based logistics are two new changes reshaping the industry .

Hertz and Alfredsson (2003) distinguish between four different kinds of 3PLs viz. standard 3PL provider (who offers the basic logistic functions, such as warehousing, distribution, pick and pack) ; service developer (who offers advanced value added services, such as cross docking, track and trace and forming specific packaging); Customer adaptor (overtaking a client's existing business and improving the efficiency in for example warehouse or logistics activities) and customer developer (who often overtakes the client's whole logistics operations, which can also be called a 'logistics integrator' or 'complexity manager') . Today, companies on average send $30 \%$ of goods through third party logistics (3PL) providers. The 3PL market is now $\$ 148$ billion in size with single-digit annual growth. ${ }^{1}$ Lai (2004) takes another classification scheme that is based on the degree of service performance and classify them as traditional freight forwarders, transformers, full service providers and nichers Following table 1 shows the activities of third party logistic service providers based on Sink et al. (1996).

The objective of the paper is to identify various metrics and then study their inter-relationship using ISM methodology. The paper is organized as follows. Section 2 deals with literature review. Section 3 explains the ISM methodology and thereafter it has been explained through case example in section 4. Finally managerial implications and directions for future research have been discussed in section 5 .

\section{LITERATURE REVIEW}

\subsection{Review of literature on use of third party logistics}

As TPLs are new to construction industry and hence it is quite obvious that suppliers and contractors do face compatibility and adjustment issues which act as barriers towards the full -fledged acceptance of TPLs in construction industry. Several exploratory studies have been performed by researchers to identify the attitudes, perceptions and behaviors of supplier's and transport providers towards TPL and their services. They have found mixed views of the respondents. Sobotka and Czarnigowska (2005) showed that when contractors outsource logistics management tasks such as handling materials, to logistics professionals, a reduction in costs could be seen. Although dedicated TPL solutions do benefit in the construction industry, it could lead to loss of source of income and control in the construction project. In either case, it is important to analyze the benefits as well as the drawbacks with the chosen solution. Therefore it is quite beneficial to examine the various metrics or traits essential for assessing and analyzing the performance of TPL. Literature has examined a variety of measures to measure general or specific performance of logistic service providers based on various traits and metrics which have been explained in detail in next section of literature review. Bygballe et al. (2010) study the interdependence in supply chains and projects in construction and found that the strong emphasis on coordination of sequential interdependence within individual supply chains does not fit with the complex interdependencies present in and among supply chains and projects in construction. Lindén and Josephson (2013) found that outsourced materials handling was advantageous compared to in-house materials handling, resulting in a cost reduction. Ekeskar et al. (2014) perform an exploratory study to investigate the use of 3PL providers in large construction projects and identify main drivers and barriers, as well as the resulting effects, when implementing SCM by the use of 3PL providers. For example, Ekeskär et al. (2014) found that by using 3PL, a structured interface between the supply chain and the construction site is established. Ekeskär et al. (2014) also identified that the time spent on materials handling decreased in favor for direct value adding tasks when a 3PL provider was employed. Ekeskar and Rudberg (2016) explore the use of a TPL provider in a large hospital project and analyze its resulting effects. The research is based on a literature review and an explorative case study of a large hospital project in Sweden, where the client and the main contractor have initiated the use of a TPL provider to coordinate sourcing and materials handling activities on site. The results show positive effects on establishing an effective interface between the construction site and the supply chain. Aggarwal \& Singh (2018) and Aggarwal (2019) presents the NPV maximization problem with a TPL and TPL selection using AHP -DEAHP approach respectively.

\subsection{Literature review on Metrics}

Third party logistics service providers could be assessed based on the performance measures such as timeliness and accuracy ( Bromley, 2001; Johnson , 2001); delivery performance (Stewart, 1995), personnel scheduling and safety measures (Mejza et al. 2003) . Similarly, Mentzer and Konrad (1991) define performance measures in five sub-areas of logistics viz. transportation, warehousing, inventory control and order processing and logistics administration. Logistic service providers can also be distinguished based on characteristics of customer relationships (Knemeyer et al. 2003) and customer satisfaction and loyalty (Stank et al. 2003). Besides this cultural traits such as communication, integrity, pro-activeness, responsiveness, process improvement and continuous improvement ${ }^{2}$ are also used for assessing TPLs in construction projects .

Nyström (2005) illustrates that trust and mutual understanding are necessary and common components and are central to all partnering and outsourcing projects. Yeung et al. (2007) has elaborated further on how the components can be interpreted and distinguish between "hard" and "soft" components. However, Karrbom Gustavsson and Hallin (2014) criticized the dichotomization of project management theories into "hard" and "soft" and indicated that components are opposite to each other. Jothimani and Sarmah (2014) proposed to apply a supply chain operations reference (SCOR) model and MCDM methods to identify KPI of 3PL companies and measure supply chain performance of 3PL companies. SCOR model considered both effectiveness and efficiency aspects of performance measurement, as well as recognizes internal and customer-related reasons for measuring performance.

As an industry evaluation of TPL industry and aspects related to finance, there are three types of metrics considered. First one is the cycle metrics which includes days of inventory or 
cash to cash cycle, days of payable (DOP), increase in days of receivables (DOR). A decrease in DOP means 3PLs are paying their bills more quickly, but the rise in DOR means this gain is nearly negated by a slower collection of monies owed to them by clients. More focused management of DOR is a possible avenue to consider in an effort to decrease the cash-to-cash cycle and improve the general cash flow management through the firms. Then there are complexity metrics which includes Revenue per employee (RPE) which is a simple measure of the monetary value each employee or third party logistic service providers provides to the business. Getting the right talent in place and creating an environment of innovative thinking and strategic relationships with clients are critical. Getting this right can significantly improve revenue without requiring excessive payroll investments.

\section{INTERPRETIVE STRUCTURAL MODELLING METHODOLOGY}

Suggested by Warfield (1994), ISM works with the following steps: it starts with identifying the relevant elements and pairwise establishing the contextual relationship amongst them. Thereafter, a structural self-interaction matrix (SSIM) may be developed between two variables i.e. $i$ and $j$ establishing a "Lead to" relationship between criteria. Four symbols viz. V, $\mathrm{A}, \mathrm{X} \& \mathrm{O}$ are used for establishing the relationships. It further lead to developing initial reachability matrix and then a final reachability matrix after removing transitivity. Afterwards, the reachability set and antecedent set for each criterion and for each element can be obtained from the final reachability matrix . After that a level partition matrix can be obtained based on establishing the precedence relationships and arranging the elements in a topological order. Finally a Mic-Mac analysis is performed categorizing the variables in to autonomous, dependent, driver and linkage category. Finally, a diagraph can be obtained.

\section{CASE EXAMPLE}

In this section, ISM model is developed for studying the interrelationships amongst various metrics for measuring the performance of third party logistic service providers in construction projects supply chains. Sixteen important criteria considered are : timeliness and accuracy (TNA); better delivery performance (BDP); personnel scheduling and safety measures (PSSM); customer satisfaction and loyalty (CSR); level of communication (CoM); Pro-activeness (PA) ; Responsiveness (Res) ; process improvement and continuous improvement (PCI); trust and mutual understanding (TMU); effectiveness of TPL (ETPL) and efficiency of TPL (EfTPL); decrease in days of inventory (DDI) ; decrease in days of payable (DDOP) ; decrease in days of receivables (DDOR) ; increase in revenue per employee (IRPE); innovative thinking and strategic relationships with clients (ITSR) .

\subsection{Construction of Structural self- interaction Matrix (SSIM)}

This matrix gives the pair-wise relationship between two variables i.e. $i$ and $j$ based on VAXO. SSIM has been presented below in Fig 1.

\subsection{Construction of Initial Reachability Matrix and final reachability matrix}

The SSIM has been converted in to a binary matrix called the initial reachability matrix shown in fig. 2 by substituting $\mathrm{V}, \mathrm{A}$, $\mathrm{X}, \mathrm{O}$ by 1 or 0 as per the case. After incorporating the transitivity, the final reachability matrix is shown below in the Fig 3.

\begin{tabular}{|c|c|c|c|c|c|c|c|c|c|c|c|c|c|c|c|c|c|}
\hline & Barrier & 1 & 2 & 3 & 4 & 5 & 6 & 7 & 8 & 9 & 10 & 11 & 12 & 13 & 14 & 15 & 16 \\
\hline & & $\begin{array}{c}\text { TN } \\
\text { A }\end{array}$ & $\begin{array}{c}\text { BD } \\
P\end{array}$ & $\begin{array}{c}\text { PSS } \\
\text { M }\end{array}$ & $\begin{array}{c}\mathrm{CS} \\
\mathrm{R}\end{array}$ & $\begin{array}{l}\mathrm{Co} \\
\mathrm{M}\end{array}$ & $\begin{array}{l}\mathrm{P} \\
\mathrm{A}\end{array}$ & $\begin{array}{c}\mathrm{Re} \\
\mathrm{s}\end{array}$ & $\begin{array}{c}\mathrm{PC} \\
\mathrm{I}\end{array}$ & $\begin{array}{c}\text { TM } \\
\text { U }\end{array}$ & $\begin{array}{c}\text { ETP } \\
\text { L }\end{array}$ & $\begin{array}{c}\text { EfTP } \\
\text { L }\end{array}$ & $\begin{array}{c}\text { DD } \\
\text { I }\end{array}$ & $\begin{array}{c}\text { DDO } \\
\text { P }\end{array}$ & $\begin{array}{c}\text { DDO } \\
\text { R }\end{array}$ & $\begin{array}{c}\text { IRP } \\
\text { E }\end{array}$ & $\begin{array}{c}\text { ITS } \\
\text { R }\end{array}$ \\
\hline 1 & TNA & & $\mathrm{V}$ & $\mathrm{V}$ & $\mathrm{V}$ & $\mathrm{O}$ & $\bar{X}$ & $\mathrm{X}$ & $\mathrm{V}$ & $\mathrm{V}$ & $\mathrm{V}$ & $\mathrm{V}$ & $\mathrm{V}$ & $\mathrm{V}$ & $\mathrm{V}$ & $\mathrm{V}$ & $\mathrm{V}$ \\
\hline 2 & BDP & & & A & V & A & A & A & A & V & V & A & V & V & V & V & $X$ \\
\hline 3 & PSSM & & & & $\mathrm{V}$ & $\mathrm{O}$ & $\mathrm{A}$ & $\mathrm{O}$ & V & V & $\mathrm{V}$ & $\mathrm{V}$ & $\mathrm{O}$ & $\mathrm{O}$ & $\mathrm{O}$ & V & $X$ \\
\hline 4 & CSR & & & & & $\mathrm{A}$ & A & A & A & V & V & V & V & V & V & V & $\mathrm{A}$ \\
\hline 5 & $\mathrm{CoM}$ & & & & & & $\mathrm{A}$ & $\mathrm{A}$ & $\mathrm{A}$ & $\mathrm{A}$ & $\mathrm{A}$ & $\mathrm{A}$ & V & V & V & V & V \\
\hline 6 & PA & & & & & & & V & V & V & V & V & V & V & V & V & V \\
\hline 7 & Res & & & & & & & & V & $\mathrm{V}$ & $\mathrm{V}$ & $\mathrm{V}$ & V & V & V & V & $\mathrm{V}$ \\
\hline 8 & PCI & & & & & & & & & V & V & V & V & V & V & V & V \\
\hline 9 & TMU & & & & & & & & & & V & V & V & V & $\mathrm{V}$ & V & V \\
\hline $\begin{array}{l}1 \\
0\end{array}$ & ETPL & & & & & & & & & & & V & V & $\mathrm{V}$ & V & V & V \\
\hline $\begin{array}{l}1 \\
1\end{array}$ & EfTPL & & & & & & & & & & & & $\mathrm{V}$ & $\mathrm{V}$ & $\mathrm{V}$ & $\mathrm{V}$ & $\mathrm{V}$ \\
\hline $\begin{array}{l}1 \\
2\end{array}$ & DDI & & & & & & & & & & & & & $\mathrm{V}$ & V & V & $\mathrm{O}$ \\
\hline $\begin{array}{l}1 \\
3\end{array}$ & DDOP & & & & & & & & & & & & & & $\mathrm{V}$ & $\mathrm{V}$ & $\mathrm{A}$ \\
\hline $\begin{array}{l}1 \\
4\end{array}$ & DDOR & & & & & & & & & & & & & & & V & A \\
\hline
\end{tabular}




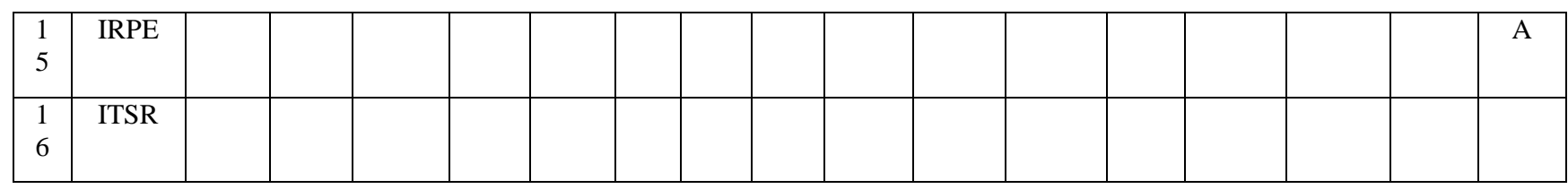

Fig 1: SSIM matrix for pair wise relationship amongst Metrics

\begin{tabular}{|c|c|c|c|c|c|c|c|c|c|c|c|c|c|c|c|c|c|}
\hline & Barriers & 1 & 2 & 3 & 4 & 5 & 6 & 7 & 8 & 9 & 10 & 11 & 12 & 13 & 14 & 15 & 16 \\
\hline & & TNA & BDP & PSSM & CSR & $\mathrm{CoM}$ & PA & Res & PCI & TMU & ETPL & EfTPL & DDI & DDOP & DDOR & IRPE & ITSR \\
\hline 1 & TNA & 1 & 1 & 1 & 1 & 0 & 1 & 1 & 1 & 1 & 1 & 1 & 1 & 1 & 1 & 1 & 1 \\
\hline 2 & BDP & 0 & 1 & 0 & 1 & 0 & 0 & 0 & 0 & 1 & 1 & 0 & 1 & 1 & 1 & 1 & 1 \\
\hline 3 & PSSM & 0 & 1 & 1 & 1 & 0 & 0 & 0 & 1 & 1 & 1 & 1 & 0 & 0 & 0 & 1 & 1 \\
\hline 4 & CSR & 0 & 0 & 0 & 1 & 0 & 0 & 0 & 0 & 1 & 1 & 1 & 1 & 1 & 1 & 1 & 0 \\
\hline 5 & $\mathrm{CoM}$ & 0 & 1 & 0 & 1 & 1 & 0 & 0 & 0 & 0 & 0 & 0 & 1 & 1 & 1 & 1 & 1 \\
\hline 6 & PA & 1 & 1 & 1 & 1 & 1 & 1 & 1 & 1 & 1 & 1 & 1 & 1 & 1 & 1 & 1 & 1 \\
\hline 7 & Res & 1 & 1 & 0 & 1 & 1 & 0 & 1 & 1 & 1 & 1 & 1 & 1 & 1 & 1 & 1 & 1 \\
\hline 8 & PCI & 0 & 1 & 0 & 1 & 1 & 0 & 0 & 1 & 1 & 1 & 1 & 1 & 1 & 1 & 1 & 1 \\
\hline 9 & TMU & 0 & 0 & 0 & 0 & 1 & 0 & 0 & 0 & 1 & 1 & 1 & 1 & 1 & 1 & 1 & 1 \\
\hline 10 & ETPL & 0 & 0 & 0 & 0 & 0 & 0 & 0 & 0 & 0 & 1 & 1 & 1 & 1 & 1 & 1 & 1 \\
\hline 11 & EfTPL & 0 & 1 & 0 & 0 & 0 & 0 & 0 & 0 & 0 & 0 & 1 & 1 & 1 & 1 & 1 & 1 \\
\hline 12 & DDI & 0 & 0 & 0 & 0 & 0 & 0 & 0 & 0 & 0 & 0 & 0 & 1 & 1 & 1 & 1 & 0 \\
\hline 13 & DDOP & 0 & 0 & 0 & 0 & 0 & 0 & 0 & 0 & 0 & 0 & 0 & 0 & 1 & 1 & 1 & 0 \\
\hline 14 & DDOR & 0 & 0 & 0 & 0 & 0 & 0 & 0 & 0 & 0 & 0 & 0 & 0 & 0 & 1 & 1 & 0 \\
\hline 15 & IRPE & 0 & 0 & 0 & 0 & 0 & 0 & 0 & 0 & 0 & 0 & 0 & 0 & 0 & 0 & 1 & 0 \\
\hline 16 & ITSR & 0 & 1 & 1 & 1 & 1 & 0 & 0 & 0 & 0 & 0 & 0 & 0 & 1 & 1 & 1 & 1 \\
\hline
\end{tabular}

Fig 2: Initial reachability matrix

\begin{tabular}{|c|c|c|c|c|c|c|c|c|c|c|c|c|c|c|c|c|c|c|}
\hline & Barriers & 1 & 2 & 3 & 4 & 5 & 6 & 7 & 8 & 9 & 10 & 11 & 12 & 13 & 14 & 15 & 16 & \\
\hline & & TNA & BDP & PSSM & CSR & CoM & PA & Res & PCI & TMU & ETPL & EfTPL & DDI & DDOP & DDOR & IRPE & ITSR & D.P \\
\hline 1 & TNA & 1 & 1 & 1 & 1 & 1 & 1 & 1 & 1 & 1 & 1 & 1 & 1 & 1 & 1 & 1 & 1 & 16 \\
\hline 3 & PSSM & 0 & 1 & 1 & 1 & 1 & 0 & 0 & 1 & 1 & 1 & 1 & 1 & 1 & 1 & 1 & 1 & 13 \\
\hline 4 & CSR & 0 & 0 & 0 & 1 & 1 & 0 & 0 & 0 & 1 & 1 & 1 & 1 & 1 & 1 & 1 & 1 & 10 \\
\hline 6 & PA & 1 & 1 & 1 & 1 & 1 & 1 & 1 & 1 & 1 & 1 & 1 & 1 & 1 & 1 & 1 & 1 & 16 \\
\hline 7 & Res & 1 & 1 & 0 & 1 & 1 & 0 & 1 & 1 & 1 & 1 & 1 & 1 & 1 & 1 & 1 & 1 & 14 \\
\hline 8 & PCI & 0 & 1 & 1 & 1 & 1 & 0 & 0 & 1 & 1 & 1 & 1 & 1 & 1 & 1 & 1 & 1 & 13 \\
\hline 9 & TMU & 0 & 1 & 0 & 1 & 1 & 0 & 0 & 0 & 1 & 1 & 1 & 1 & 1 & 1 & 1 & 1 & 11 \\
\hline 10 & ETPL & 0 & 0 & 0 & 0 & 0 & 0 & 0 & 0 & 0 & 1 & 1 & 1 & 1 & 1 & 1 & 1 & 7 \\
\hline 14 & DDOR & 0 & 0 & 0 & 0 & 0 & 0 & 0 & 0 & 0 & 0 & 0 & 0 & 0 & 1 & 1 & 0 & 2 \\
\hline 15 & IRPE & 0 & 0 & 0 & 0 & 0 & 0 & 0 & 0 & 0 & 0 & 0 & 0 & 0 & 0 & 1 & 0 & 1 \\
\hline \multirow[t]{2}{*}{16} & ITSR & 0 & 1 & 1 & 1 & 1 & 0 & 0 & 0 & 0 & 0 & 0 & 0 & 1 & 1 & 1 & 1 & 8 \\
\hline & De.P & 3 & 10 & 5 & 10 & 10 & 2 & 3 & 5 & 9 & 10 & 11 & 12 & 14 & 15 & 16 & 12 & \\
\hline
\end{tabular}

Fig 3 : Final reachability matrix

D.P : Driving power ; De.P : dependence power 


\subsection{Level Partition}

From the final reachability matrix, reachability and final antecedent set for each factor are found. The elements for which the reachability and intersection sets are same are the top-level element in the ISM hierarchy. After the identification of top level element, it is separated out from the other elements and the process continues for next level of elements. Reachability set, antecedent set, intersection set along with different level for elements have been shown below in table 4.3.1.

Table 4.3.1: Iteration I

\begin{tabular}{|c|c|c|c|c|}
\hline $\begin{array}{l}\text { S. } \\
\text { No } \\
\text {. }\end{array}$ & $\begin{array}{c}\text { Reachability } \\
\text { set }\end{array}$ & $\begin{array}{c}\text { Antecedent } \\
\text { set }\end{array}$ & $\begin{array}{c}\text { Intersect } \\
\text { ion set }\end{array}$ & Level \\
\hline 1. & 15 & $\begin{array}{c}1,2,3,4,5,67,8 \\
9,10,11,12,1,3 \\
, 14,15,16\end{array}$ & 15 & \multirow{12}{*}{ I } \\
\hline 2. & 14,15 & $\begin{array}{c}1,2,3,4,5,6,7,8 \\
, 9,10,11,12,13 \\
, 14,16\end{array}$ & 14 & \\
\hline 3. & $13,14,15$ & $\begin{array}{c}1,2,3,4,5,6,7,8 \\
, 9,10,11,12,13 \\
, 16\end{array}$ & 13 & \\
\hline 4. & $\begin{array}{c}13,14,15 \\
16\end{array}$ & $\begin{array}{c}1,2,3,4,5,6,7,8 \\
, 9,10,11,16\end{array}$ & 16 & \\
\hline 5. & $\begin{array}{c}12,13,14 \\
15\end{array}$ & $\begin{array}{l}1,2,3,4,5,6,7,8 \\
, 9,10,11,12,13\end{array}$ & 12 & \\
\hline 6. & $\begin{array}{c}11,12,13,14 \\
15,16\end{array}$ & $\begin{array}{c}1,2,3,4,5,6,7,8 \\
, 9,10,11\end{array}$ & 11 & \\
\hline 7. & $\begin{array}{c}10,11,12,13 \\
14,15,16\end{array}$ & $\begin{array}{c}1,2,3,4,5,6,7,8 \\
, 9,10\end{array}$ & 10 & \\
\hline 8. & $\begin{array}{l}2,10,11,12, \\
13,14,15,16\end{array}$ & $1,2,3,5,6,7,8,9$ & 2 & \\
\hline 9 & $\begin{array}{l}4,10,11,12, \\
13,14,15,16\end{array}$ & $\begin{array}{c}1,2,3,4,5,6,7,8 \\
, 9\end{array}$ & 4 & \\
\hline 10 & $\begin{array}{c}2,4,5,9,10 \\
11,12,13,14 \\
15,16\end{array}$ & $1,2,3,5,6,7,8,9$ & $2,5,9$ & \\
\hline 11 & $\begin{array}{c}2,3,4,5,8,9 \\
10,11,12,13 \\
14,15,16\end{array}$ & $1,2,3,5,6,7,8,9$ & $2,3,5,8,9$ & \\
\hline 12 & $\begin{array}{c}1,2,3,4,5,7,8 \\
9,10,11,12,1 \\
3,14,15,16\end{array}$ & $1,6,7$ & 1,7 & \\
\hline 13 & $\begin{array}{c}1,2,3,4,5,6,7,8 \\
10,11,12,13,14 \\
5,16\end{array}$ & 9 & 1,6 & \\
\hline
\end{tabular}

Table 4.3.2: Iteration II

\begin{tabular}{|c|c|c|c|c|}
\hline $\begin{array}{l}\text { S. } \\
\text { No }\end{array}$ & $\begin{array}{c}\text { Reachability } \\
\text { set }\end{array}$ & $\begin{array}{c}\text { Antecedent } \\
\text { set }\end{array}$ & $\begin{array}{c}\text { Intersect } \\
\text { ion set }\end{array}$ & Level \\
\hline 2. & 14 & $\begin{array}{c}1,2,3,4,5,6,7,8 \\
, 9,10,11,13,14 \\
, 16\end{array}$ & 14 & \multirow{12}{*}{ II } \\
\hline 3. & 13,14 & $\begin{array}{c}1,2,3,4,5,6,7,8 \\
, 9,10,11,12,13 \\
, 16\end{array}$ & 13,14 & \\
\hline 4. & $13,14,16$ & $\begin{array}{c}1,2,3,4,5,6,7,8 \\
, 9,10,11,16\end{array}$ & $13,14,16$ & \\
\hline 5. & $12,13,14$ & $\begin{array}{l}1,2,3,4,5,6,7,8 \\
, 9,10,11,12,13\end{array}$ & $12,13,14$ & \\
\hline 6. & $\begin{array}{c}11,12,13,14, \\
16\end{array}$ & $\begin{array}{c}1,2,3,4,5,6,7,8 \\
, 9,10,11\end{array}$ & 11 & \\
\hline 7. & $\begin{array}{c}10,11,12,13, \\
14,16\end{array}$ & $\begin{array}{c}1,2,3,4,5,6,7,8 \\
, 9,10\end{array}$ & 10 & \\
\hline 8. & $\begin{array}{c}2,10,11,12, \\
13,14,16\end{array}$ & $1,2,3,5,6,7,8,9$ & 2 & \\
\hline 9 & $\begin{array}{c}4,10,11,12, \\
13,14,16\end{array}$ & $\begin{array}{c}1,2,3,4,5,6,7,8 \\
, 9\end{array}$ & 4 & \\
\hline 10 & $\begin{array}{c}2,4,5,9,10, \\
11,12,13,14, \\
16\end{array}$ & $1,2,3,5,6,7,8,9$ & $2,5,9$ & \\
\hline 11 & $\begin{array}{c}2,3,4,5,8,9, \\
10,11,12,13, \\
14,16\end{array}$ & $1,2,3,5,6,7,8,9$ & $2,3,5,8,9$ & \\
\hline 12 & $\begin{array}{c}1,2,3,4,5,7,8, \\
9,10,11,12,1 \\
3,14,16\end{array}$ & $1,6,7$ & 1,7 & \\
\hline 13 & $\begin{array}{c}1,2,3,4,5,6,7, \\
8,9,10,11, \\
12,13,14,16\end{array}$ & 1,6 & 1,6 & \\
\hline
\end{tabular}

Table 4.3.3: Iteration III

\begin{tabular}{|c|c|c|c|c|}
\hline $\begin{array}{l}\text { S. } \\
\text { No. }\end{array}$ & $\begin{array}{c}\text { Reachability } \\
\text { set }\end{array}$ & $\begin{array}{l}\text { Antecedent } \\
\text { set }\end{array}$ & $\begin{array}{c}\text { Intersect } \\
\text { ion set }\end{array}$ & Level \\
\hline 3. & 13 & $\begin{array}{c}1,2,3,4,5,6,7,8 \\
9,10,11,12,13 \\
, 16\end{array}$ & 13 & \\
\hline 4. & 13,16 & $\begin{array}{c}1,2,3,4,5,6,7,8 \\
, 9,10,11,16\end{array}$ & 13,16 & \\
\hline 5. & 12,13 & $\begin{array}{l}1,2,3,4,5,6,7,8 \\
, 9,10,11,12,13\end{array}$ & 12,13 & \\
\hline 6. & $11,12,13,16$ & $\begin{array}{c}1,2,3,4,5,6,7,8 \\
, 9,10,11\end{array}$ & 11 & \\
\hline 7. & $\begin{array}{c}10,11,12,13 \\
16\end{array}$ & $\begin{array}{c}1,2,3,4,5,6,7,8 \\
, 9,10\end{array}$ & 10 & \\
\hline 8. & $2,10,11,12$ & $1,2,3,5,6,7,8,9$ & 2 & \\
\hline
\end{tabular}




\begin{tabular}{|c|c|c|c|c|}
\hline & 13,16 & & & \\
\hline 9 & $\begin{array}{c}4,10,11,12 \\
13,16\end{array}$ & $\begin{array}{c}1,2,3,4,5,6,7,8 \\
, 9\end{array}$ & 4 & \\
\hline 10 & $\begin{array}{c}2,4,5,9,10 \\
11,12,13,16\end{array}$ & $1,2,3,5,6,7,8,9$ & $2,5,9$ & \\
\hline 11 & $\begin{array}{c}2,3,4,5,8,9 \\
10,11,12,13 \\
16\end{array}$ & $1,2,3,5,6,7,8,9$ & $2,3,5,8,9$ & \\
\hline 12 & $\begin{array}{c}1,2,3,4,5,7,8 \\
9,10,11,12,1 \\
3,16\end{array}$ & $1,6,7$ & 1,7 & III \\
\hline 13 & $\begin{array}{c}1,2,3,4,5,6,7 \\
8,9,10,11 \\
12,13,16\end{array}$ & 1,6 & 1,6 & \\
\hline
\end{tabular}

Table 4.3.4: Iteration IV

\begin{tabular}{|c|c|c|c|c|}
\hline $\begin{array}{l}\text { S. } \\
\text { No }\end{array}$ & $\begin{array}{c}\text { Reachability } \\
\text { set }\end{array}$ & $\begin{array}{c}\text { Antecedent } \\
\text { set }\end{array}$ & $\begin{array}{c}\text { Intersect } \\
\text { ion set }\end{array}$ & Level \\
\hline 4. & 16 & $\begin{array}{c}1,2,3,4,5,6,7,8 \\
, 9,10,11,16\end{array}$ & 16 & \multirow{11}{*}{ IV } \\
\hline 5. & 12 & $\begin{array}{c}1,2,3,4,5,6,7,8 \\
, 9,10,11,12\end{array}$ & 12 & \\
\hline 6. & $11,12,16$ & $\begin{array}{c}1,2,3,4,5,6,7,8 \\
, 9,10,11\end{array}$ & 11 & \\
\hline 7. & $10,11,12,16$ & $\begin{array}{c}1,2,3,4,5,6,7,8 \\
, 9,10\end{array}$ & 10 & \\
\hline 8. & $2,10,11,12,16$ & $1,2,3,5,6,7,8,9$ & 2 & \\
\hline 9 & $4,10,11,12,16$ & $\begin{array}{c}1,2,3,4,5,6,7,8 \\
, 9\end{array}$ & 4 & \\
\hline 10 & $\begin{array}{c}2,4,5,9,10,11,1 \\
2,16\end{array}$ & $1,2,3,5,6,7,8,9$ & $2,5,9$ & \\
\hline 11 & $\begin{array}{c}2,3,4,5,8,9,10 \\
11,12,16\end{array}$ & $1,2,3,5,6,7,8,9$ & $2,3,5,8,9$ & \\
\hline 12 & $\begin{array}{c}1,2,3,4,5,7,8,9 \\
10,11,12,16\end{array}$ & $1,6,7$ & 1,7 & \\
\hline 13 & $\begin{array}{c}1,2,3,4,5,6,7,8 \\
9,10,11,12,16\end{array}$ & 1,6 & 1,6 & \\
\hline 4. & 16 & $\begin{array}{c}1,2,3,4,5,6,7,8 \\
, 9,10,11,16\end{array}$ & 16 & \\
\hline
\end{tabular}

Table 4.3.5: Iteration V

\begin{tabular}{|c|c|c|c|c|}
\hline $\begin{array}{l}\text { S. } \\
\text { No. }\end{array}$ & $\begin{array}{c}\text { Reachability } \\
\text { set }\end{array}$ & $\begin{array}{c}\text { Antecedent } \\
\text { set }\end{array}$ & $\begin{array}{c}\text { Intersect } \\
\text { ion set }\end{array}$ & Level \\
\hline 6. & 11 & $\begin{array}{c}1,2,3,4,5,6,7,8 \\
, 9,10,11\end{array}$ & 11 & \multirow{8}{*}{$\mathbf{V}$} \\
\hline 7. & 10,11 & $\begin{array}{c}1,2,3,4,5,6,7,8 \\
, 9,10\end{array}$ & 10 & \\
\hline 8. & $2,10,11$ & $1,2,3,5,6,7,8,9$ & 2 & \\
\hline 9 & $4,10,11$ & $\begin{array}{c}1,2,3,4,5,6,7,8 \\
, 9\end{array}$ & 4 & \\
\hline 10 & $\begin{array}{c}2,4,5,9,10, \\
11\end{array}$ & $1,2,3,5,6,7,8,9$ & $2,5,9$ & \\
\hline 11 & $\begin{array}{c}2,3,4,5,8,9, \\
10,11\end{array}$ & $1,2,3,5,6,7,8,9$ & $2,3,5,8,9$ & \\
\hline 12 & $\begin{array}{c}1,2,3,4,5,7,8 \\
9,10,11\end{array}$ & $1,6,7$ & 1,7 & \\
\hline 13 & $\begin{array}{c}1,2,3,4,5,6,7 \\
8,9,10,11\end{array}$ & 1,6 & 1,6 & \\
\hline
\end{tabular}

Table 4.3.6: Iteration VI

\begin{tabular}{|c|c|c|c|c|}
\hline $\begin{array}{l}\text { S. } \\
\text { No. }\end{array}$ & $\begin{array}{c}\text { Reachability } \\
\text { set }\end{array}$ & $\begin{array}{c}\text { Antecedent } \\
\text { set }\end{array}$ & $\begin{array}{c}\text { Intersect } \\
\text { ion set }\end{array}$ & Level \\
\hline 7. & 10 & $\begin{array}{c}1,2,3,4,5,6,7,8 \\
, 9,10\end{array}$ & 10 & \\
\hline 8. & 2,10 & $1,2,3,5,6,7,8,9$ & 2 & \\
\hline 9 & 4,10 & $\begin{array}{c}1,2,3,4,5,6,7,8 \\
, 9\end{array}$ & 4 & \\
\hline 10 & $2,4,5,9,10$ & $1,2,3,5,6,7,8,9$ & $2,5,9$ & \\
\hline 11 & $\begin{array}{c}2,3,4,5,8,9 \\
10\end{array}$ & $1,2,3,5,6,7,8,9$ & $2,3,5,8,9$ & \\
\hline 12 & $\begin{array}{c}1,2,3,4,5,7,8 \\
9,10\end{array}$ & $1,6,7$ & 1,7 & \\
\hline 13 & $\begin{array}{c}1,2,3,4,5,6,7 \\
8,9,10\end{array}$ & 1,6 & 1,6 & VI \\
\hline
\end{tabular}


Table 4.3.7: Iteration VII

\begin{tabular}{|c|c|c|c|c|}
\hline $\begin{array}{l}\text { S. } \\
\text { No. }\end{array}$ & $\begin{array}{c}\text { Reachability } \\
\text { set }\end{array}$ & $\begin{array}{l}\text { Antecedent } \\
\text { set }\end{array}$ & $\begin{array}{c}\text { Intersection } \\
\text { set }\end{array}$ & Level \\
\hline 8. & 2 & $\begin{array}{c}1,2,3,5,6,7,8 \\
9\end{array}$ & 2 & \multirow{6}{*}{ VII } \\
\hline 9 & 4 & $\begin{array}{c}1,2,3,4,5,6,7 \\
8,9\end{array}$ & 4 & \\
\hline 10 & $2,4,5,9$ & $\begin{array}{c}1,2,3,5,6,7,8 \\
9\end{array}$ & $2,5,9$ & \\
\hline 11 & $2,3,4,5,8,9$ & $\begin{array}{c}1,2,3,5,6,7,8 \\
9\end{array}$ & $2,3,5,8,9$ & \\
\hline 12 & $\begin{array}{c}1,2,3,4,5,7,8 \\
9\end{array}$ & $1,6,7$ & 1,7 & \\
\hline 13 & $\begin{array}{c}1,2,3,4,5,6,7 \\
8,9\end{array}$ & 1,6 & 1,6 & \\
\hline
\end{tabular}

Table 4.3.8: Iteration VIII

\begin{tabular}{|c|c|c|c|c|}
\hline $\begin{array}{c}\text { S. } \\
\text { No. }\end{array}$ & $\begin{array}{c}\text { Reachability } \\
\text { set }\end{array}$ & $\begin{array}{c}\text { Antecedent } \\
\text { set }\end{array}$ & $\begin{array}{c}\text { Intersectio } \\
\text { n set }\end{array}$ & Level \\
\hline 8. & 2 & $1,2,3,5,6,7,8,9$ & 2 & \\
\hline 10 & $\mathbf{2 , 5 , 9}$ & $1,2,3,5,6,7,8,9$ & $2,5,9$ & \multirow{2}{*}{ VIII } \\
\hline 11 & $2,3,5,8,9$ & $1,3,6,7,8,9$ & $3,8,9$ & \\
\hline 12 & $1,2,3,5,7,8,9$ & $1,6,7$ & 1,7 & \\
\hline 13 & $1,2,3,5,6,7,8,9$ & 1,6 & 1,6 & \\
& & & & \\
& & & & \\
\end{tabular}

Table 4.3.9: Iteration IX

\begin{tabular}{|c|c|c|c|c|}
\hline $\begin{array}{c}\text { S. } \\
\text { No. }\end{array}$ & $\begin{array}{c}\text { Reachability } \\
\text { set }\end{array}$ & $\begin{array}{c}\text { Antecedent } \\
\text { set }\end{array}$ & $\begin{array}{c}\text { Intersect } \\
\text { ion set }\end{array}$ & Level \\
\hline 11 & $\mathbf{3 , 8}$ & $1,3,6,7,8$ & 3,8 & \multirow{2}{*}{ IX } \\
\hline 12 & $1,3,7,8$ & $1,6,7$ & 1,7 & \\
\cline { 1 - 4 } 13 & $1,3,6,7,8$ & 1,6 & 1,6 & \\
\hline
\end{tabular}

Table 4.3.10: Iteration $X$

\begin{tabular}{|c|c|c|c|c|}
\hline $\begin{array}{c}\text { S. } \\
\text { No. }\end{array}$ & $\begin{array}{c}\text { Reachability } \\
\text { set }\end{array}$ & $\begin{array}{c}\text { Antecedent } \\
\text { set }\end{array}$ & $\begin{array}{c}\text { Intersect } \\
\text { ion set }\end{array}$ & Level \\
\hline 12 & 1,7 & $1,6,7$ & 1,7 & \\
\hline 13 & $1,6,7$ & 1,6 & 1,6 & \\
\hline
\end{tabular}

\subsection{Driving power and dominance diagram}

As we can see that there is no autonomous criteria. Criteria TMU , CoM , CSR , BDP and ETPL are linkage criteria whereas criteria PA, TNA Res, PSSM and PCI are driver criteria .

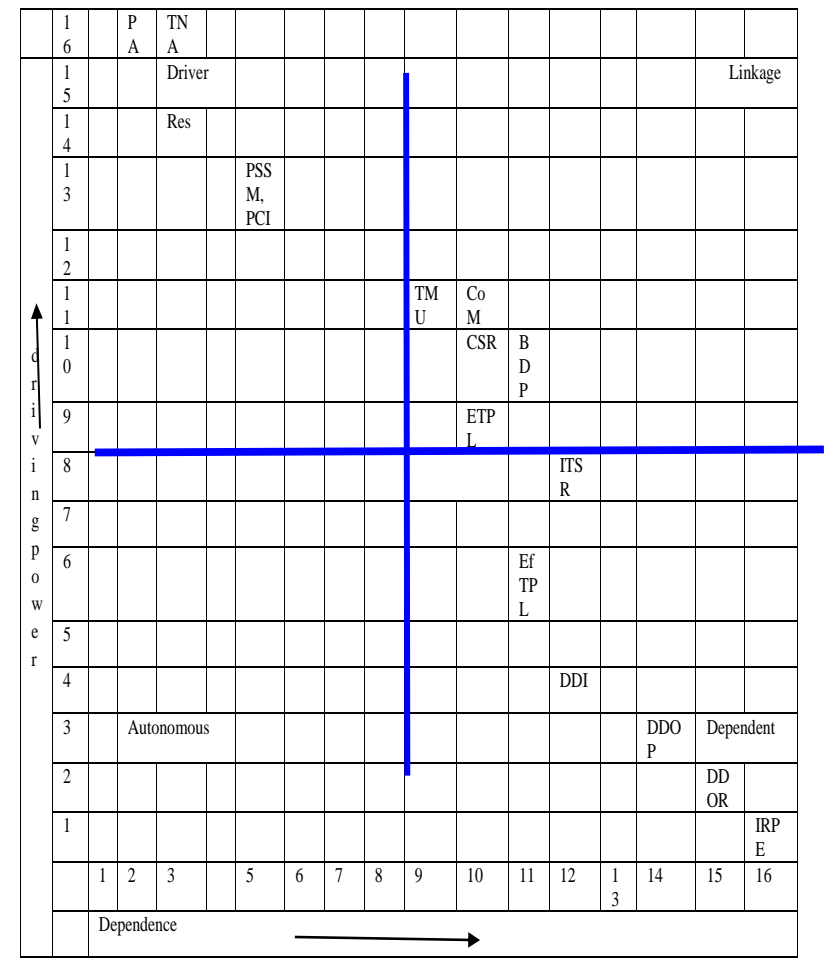

4.5 ISM --The Diagraph

The diagraph presenting the hierarchy of the various performance metrics is shown in figure below: 


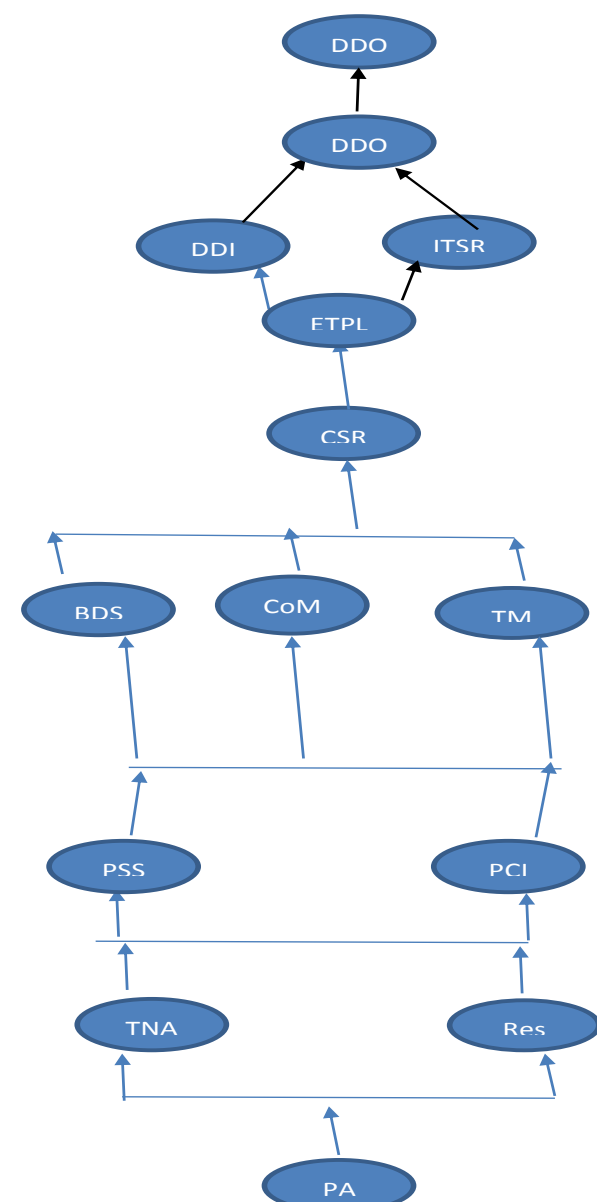

Figure : Diagraph for various performance metrics for TPL

\section{REFERENCES}

[1] Abdel-Wahab, M. and Vogl, B. 2011. Trends of productivity growth in the construction industry across Europe, US and Japan, Construction Management and Economics, 29(6), 635-644.

[2] Aggarwal , R. and Singh, S.P. 2019 . An integrated NPV based supply chain configuration with third party logistics services, Journal of Revenue and Pricing Management, Springer.

[3] Aggarwal , R. 2019. Third Party Logistics Service Providers Selection using AHP-DEAHP approach. in International Journal of integrated supply management (IJISM ), Inderscience Publishers.

[4] Aggarwal, R. and Singh, S. 2015. RDEAHP approach for supplier selection. IJOR , Inderscience Publishers.

[5] Bankvall, L., Bygballe, L. E., Dubois, A. and Jahre, M. 2010. Interdependence in supply chains and projects in construction. Supply Chain Management, 15(55), 385393.

[6] Vrijhoef, R. and London, K. A. (eds.) Construction Supply Chain Management Handbook, CRC Press, 12-112-22.

[7] Dubois, A. and Gadde, L.-E. 2002. The construction industry as a loosely coupled system: implications for productivity and innovation, Construction Management \& Economics, 20 (7) ,621-631.

[8] Ekeskär, A., Rudberg, M. \& Vennström, A. 2014. Third- party logistics in large construction projects: A SCM perspective, paper presented at 21st International Annual EurOMA Conference, Palermo, 20-25 June.

[9] Mentzer, J. T., Dewitt, W., Keebler, J. S., Min, S., Nix, N. W., Smith, C. D. and Zacharia, Z. G. 2001. Defining supply chain management, Journal of Business Logistics, 22(2), 1-25.

[10] Miller, C. J. M., Packham, G. A. and Thomas, B. C. 2002. Harmonization between Main Contractors and Subcontractors: A Prerequisite for Lean Construction?, Journal of Construction Research, 3(1), 67-82.

[11] Sobotka, A. and Czarnigowska, A. 2005. Analysis of supply system models for planning construction project logistics, Journal of Civil Engineering and Management, 11(1), 73-82.

[12] Vidalakis, C. and Sommerville, J. 2013. Transportation responsiveness and efficiency within the building supply chain, Building Research \& Information, 41(4), 469-481.

[13] Vrijhoef, R. and Koskela, L. 2000. The four roles of supply chain management in construction, European Journal of Purchasing \& Supply Management, 6(3-4), 169-178.

[14] Agapiou, A., Flanagan, R., Norman, G. and Notman, D. 1998b. The changing role of builders merchants in the construction supply chain. Construction Management and Economics, 16, 351-361.

[15] Aguezzoul, Aicha 2014. Third-party logistics selection problem: A literature review on criteria and methods. Omega, 49, 69-78.

[16] Akintoye, A., McIntosh, G. \& Fitzgerald, E. 2000. A survey of supply chain collaboration and management in the UK construction industry. European Journal of Purchasing and Supply Management, 6, 159-168.

[17] Bankvall, Lars, Bygballe, Lena E., Dubois, Anna \& Jahre, Marianne 2010. Interdependence in supply chains and projects in construction. Supply Chain Management, $15,385-393$.

[18] Behera, P., Mohanty, R. P. and Anand, P. 2015 Understanding Construction Supply Chain Management. Production Planning \& Control, 26, 1332-1350.

[19] Liu, C.L. and Lyons, A.C. 2011. An analysis of thirdparty logistics performance and service provision. Transportation Research Part E: Logistics and Transportation Review, 47, 547-570.

[20] Odesola, I. A. 2015. Construction Labour Productivity as a Correlate of Project Performance: An Empirical Evidence for Wall Plastering Activity. Civil Engineering Dimension, 17, 1-10.

[21] Segerstedt, A. and Olofsson, T. 2010. Supply chains in the construction industry. Supply Chain Management: An International Journal, 15, 347-353.

[22] Selviaridis, K. and Spring, M. 2007. Third party logistics: a literature review and research agenda. The International Journal of Logistics Management, 18, 125150 .

[23] Sobotka, A. and Czarnigowska, A. 2005. Analysis of supply system models for planning construction project logistics. Journal of Civil Engineering and Management, 
$11,73-82$.

[24] Vrijhoef, R. and Koskela, L. 2000. The four roles of supply chain management in construction. European Journal of Purchasing \& Supply Management, 6, 169178.

[25] Miller, C.J., Thomas, B. and Packham, G. 2002. Harmonization between main contractors and subcontractors: A prerequisite for lean construction?, Journal of Construction Research .

[26] Sommerville, J. and Vidalakis , C. 2012 Transportation responsiveness and efficiency within the building supply chain, Build Res Inf.

[27] Sobotka , A. and Czarnigowska , A. 2005. Analysis of supply system models for planning construction project logistics, Journal of Civil Engineering and Management.

[28] Ekeskär, A. Vennström, A. and Rudberg , M. 2014. Third-party logistics in large construction projects: A SCM perspective, conference paper.

[29] Ekeskär , A. and Rudberg, M. 2016a. Third-party logistics in construction: the case of a large hospital project , Construction Management and Economics, 118.

[30] Jothimani , D. Sarmah , S.P. 2014. Supply Chain Performance Measurement of Third Party Logistics , Benchmarking An International Journal 21(6), 944-963.

\section{DOI: 10.1108/BIJ-09-2012-0064}

[31] Hertz, S. \& Alfredsson, M. 2003. Strategic development of third party logistics providers. Industrial Marketing Management, 32, 139-149.

[32] Lai, K-H. 2004. Service capability and performance of logistics service providers. Transportation Research Part E, 40, 385-399.

[33] Petersson, P. \& Zantvoort, T.R. 2012. Fourth-party logistics: A case study on performance measurement. Master Thesis, Jönköping International Business School.

[34] Roberti, J., Tookey, J., and Ying, J. F. 2013. SCM competencies in construction: issues and challenges in New Zealand. Journal of Engineering, Design and Technology, 13(4):522-538.

[35] Dörnhöfer, M., Schröder, F., and Günthner, W. A. 2016. Logistics performance measurement system for the automotive industry. Logistics Research, 9(1):1-26.

[36] Thunberg, M. and Persson , F. 2014. Using the SCOR model's performance measurements to improve construction logistics. Production Planning \& Control, 13-14:1065-1078.

[37] Warfield, J. N. 1974. Developing interconnection matrices in structural modeling. IEEE Transactions on System, Man, and Cybernetics, SMC-4 (1), 81-87. 